\title{
Electronic and structural properties of alkali doped SWNT
}

\author{
N. M. Nemes*, J. E. Fischer*, K. Kamarás ${ }^{\dagger}$, D. B. Tanner** and A. G. \\ Rinzler** \\ ${ }^{*}$ Laboratory on the Research of the Structure of Matter \\ University of Pennsylvania, Philadelphia, PA 19104 \\ ${ }^{\dagger}$ Research Institute for Solid State Physics and Optics \\ Hungarian Academy of Sciences, P. O. Box 49, Budapest, Hungary, H 1525 \\ ${ }^{* *}$ Department of Physics, University of Florida \\ Gainesville, FL 32611
}

\begin{abstract}
Comprehensive experiments on structural and transport properties of alkali intercalated single walled carbon nanotubes (SWNT) are presented. The increasing electron density was measured as a shift of the Drude-edge in optical reflectivity in-situ with progressive doping. In saturation-doped samples the Drude-edge shifts into the visible (to $25,000-30,000 \mathrm{~cm}-1$ for potassium and rubidium doped samples) and the samples have a golden-brown color, similar to stage I graphite. X-ray diffraction reveals a crystalline rope structure with expanded lattice constant, similar to results of Duclaux et al. . The change in the low temperature divergence of the resistivity after degassing at high temperature and high vacuum and after $\mathrm{K}$-doping is studied in-situ.
\end{abstract}

Alkali doped single walled carbon nanotubes (SWNT) have been the focus of recent research. The main picture that emerges is that the alkali dopants are located between single SWNTs within the bundles or ropes and each donates an electron to the SWNT host as is well established for graphite, polyacetylene or $\mathrm{C}_{60}$. Many important experiments have been published on alkali doped SWNT, including resistivity[1,2], Raman spectroscopy [3, 4, 5, 6], EELS[7], structural data from x-ray diffraction (XRD) [8, 9] IR and visible optical spectroscopy $[10,11,12]$. Our main finding in this report is that careful doping with alkali vapour yields SWNT with colors similar to graphite intercalation compounds (GIC). Surprisingly, this has not been reported before. There has also been a controversy over whether the 2D crystallinity of the rope lattice is destroyed upon alkali doping. We reported a loss of crystallinity in electrochemically $\mathrm{K}$ or Li doped samples[13], similar to reports on alkali-vapour doped SWNT structure[8], while the Orleans group reported an expanded rope lattice observed with XRD[9] and Pichler $e t$ al observed a shift in the zero-loss peaks in EELS[7]. We repeated the XRD experiments on vapour-doped SWNT samples and find that the rope lattice expands as shown by the shift of the XRD peaks.

For the in-situ reflectivity measurements we used carefully purified, well annealed and well characterized 'buckypapers' [14]. Small pieces were placed in 4mm EPR quartz tubes and degassed at $600-800^{\circ} \mathrm{C}$ at a base-pressure of $\approx 10^{-7}$ torr for one day or longer, a procedure which is reported to remove adsorbed oxygen[15, 16]. Then alkali metal $(\mathrm{K}$, $\mathrm{Rb}$ or $\mathrm{Cs}$ ) was distilled into the tubes without exposing the sample to ambient. For doping, the SWNT were kept at $200^{\circ} \mathrm{C}$ and carefully controlled temperature gradients were successively established, always keeping the buckypapers hotter than the alkali, ensur-

CP633, Structural and Electronic Properties of Molecular Nanostructures, edited by H. Kuzmany et al. (C) 2002 American Institute of Physics 0-7354-0088-1/02/\$19.00 

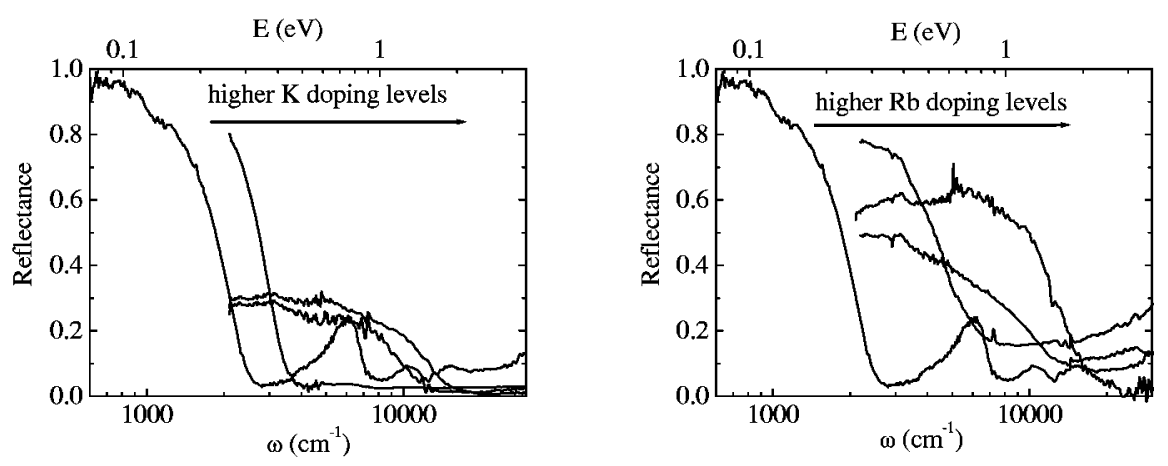

FIGURE 1. Reflectivity vs. photon energy. Left Potassium, right Rubidium. The four data sets for each sample correspond to undoped and to three different doping levels, established by consecutively smaller gradients of $100^{\circ} \mathrm{C}, 50^{\circ} \mathrm{C}, 5^{\circ} \mathrm{C}$.

ing that the samples were not coated with metal. This temperature gradient provided our control over the stoichiometry but we had no means to measure alkali vs. carbon ratios. We can only say that decreasing the gradient gave monotonically increasing alkali concentration.

We measured the reflectivity in the $0.3 \mathrm{eV}-4 \mathrm{eV}$ range of several buckypapers doped with $\mathrm{K}, \mathrm{Rb}$ or $\mathrm{Cs}$ using overlapping spectrometers. The reflectivity was normalized to that of an aluminum coated buckypaper to account for the porosity of the surface and specular reflection, following the standard method[17]. We observed the same trend in all samples as depicted in Figure $1 \mathrm{a}$ for a $\mathrm{K}$ doped and in Figure $1 \mathrm{~b}$ for $\mathrm{a} \mathrm{Rb}$ doped sample. The characteristic features in the reflectivity of the undoped sample at $0.8 \mathrm{eV}, 1.4 \mathrm{eV}$ and $2 \mathrm{eV}$, corresponding to transitions between the filled and unfilled 1D Van Hove singularities of an ensemble of SWNTs, disappear even for the lightest doping concentration. Also, the Drude edge shifts progressively to higher energies. This behaviour can be understood in light of electrochemical doping experiments[12, 11] where the stoichiometry can be controlled on a much finer scale and the different singularities can be progressively filled. On the other hand, at least for the case of $\mathrm{K}$ doping, only the vapor phase method leads to maximum K/C ratios comparable to GIC's and fullerides. For K-doping we estimate the sample composition to be $\mathrm{KC}_{8 \pm 1}$ and for $\mathrm{Rb}$ to be $\mathrm{RbC}_{5 \pm 0.5}$ based on weight uptake. The large charge transfer is manifested by the considerable Drude-edge shift. This readily explains the visible color of the samples, similar to alkali GIC's.

We observe a series of colors for $\mathrm{Rb}$ doping: using $\approx 100^{\circ} \mathrm{C}$ gradient we reproducibly achieve purple, for $\approx 50^{\circ} \mathrm{C}$ gradient golden-brown, and for $\approx 10^{\circ} \mathrm{C}$ gradient bright-gold. With Cs doping we achieve similar colors though the control is more difficult due to the large vapor pressure. With $\mathrm{K}$ we typically obtain deep red/brown samples.

The quartz sample holder prevented us from measuring below $0.3 \mathrm{eV}$. However, at low energy the highly doped samples showed reflectivity considerably less than 1 , even after normalizing for specular reflection. At present, we do not understand this but 

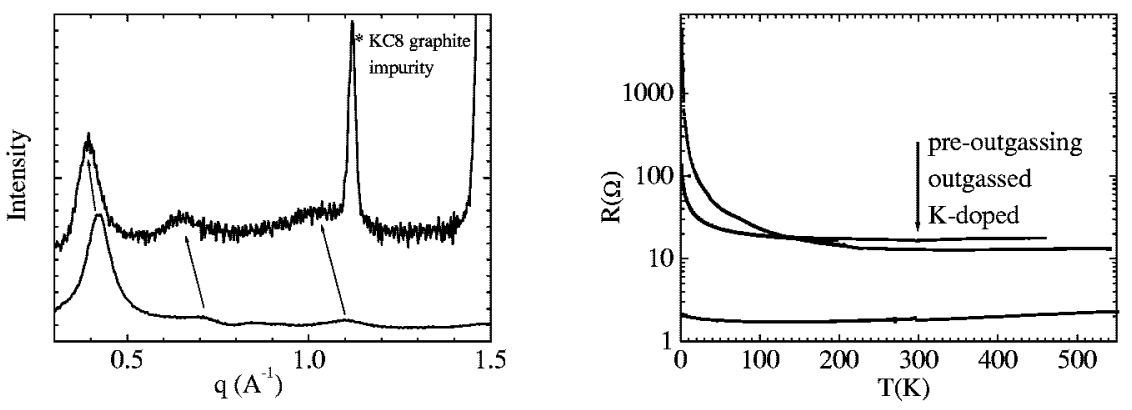

FIGURE 2. a: X-ray counts vs. wave-vector on undoped and K-doped SWNT powder. There is a clear shift to lower $\mathrm{q}$ and broadening of the peaks in the doped sample. b: Resistivity vs. temperature on the same sample, in ambient (1 year in drawer), after thorough degassing and after partial $\mathrm{K}$ doping.

experiments are under way to measure the reflectivity down to $0.07 \mathrm{eV}$. The reflectivity for the highly doped samples is flat for a large range of energies and this encourages us to ascribe the observed edge as the Drude-edge.

We also doped a piece of 'peapod' buckypaper provided by B. C. Satishkumar and D. Luzzi. We placed three samples in the same doping cell: filled peapod and empty control cut from the same piece of acid-etched unannealed buckypaper, and a piece of the well-purified/annealed buckypaper described above. All three were outgassed and doped with Cs simultaneously. Both unfilled samples achieved the usual vivid golden color whereas the filled peapod sample was distinctly darker and deep red in hue. This shows that the 'coloring' of buckypapers upon doping is a generic property, requiring only careful outgassing and vapor doping to observe the effect. The color difference is most likely due to differences in interband spectra rather than Drude plasma frequencies [18].

Samples for X-ray diffraction (XRD) were doped similarly, using SWNT powder and a gradient of $40^{\circ} \mathrm{C}$ to achieve an intermediate phase. The doped powder was loosely placed in a $2 \mathrm{~mm}$ quartz capillary. Using XRD we resolved the differing claims of our group and the Orleans-group concerning the lattice expansion of the ropes upon alkali doping. As shown in Figure 2a, intermediate K doped SWNT shows 2D rope peaks shifted to lower q-values, as already reported by Duclaux et al[9]. This sample had a dark-red color, similar to powders of $\mathrm{KC}_{8}$ stage I GIC. We can use the impurity-graphite reflections as internal reference. The sample has a mixture of stage I GIC and higher stages indicating incomplete doping. Thus it is likely that the SWNT ropes were not doped fully either. Besides the lower-q position of the peaks we observe broader reflections, indicating either a reduction in particle size or partial loss of coherence within a particle (i.e. rope). This may be explained by a doping picture where neighboring segments of each rope are either fully doped or pristine, thus the expanded and original lattices coexist on a few-nm length scale. This picture was first presented based on insitu electrochemical CESR measurements[13]. 
The resistivity was measured in-situ using a special assembly developed by Arthur F. Hebard for $\mathrm{K}_{3} \mathrm{C}_{60}$ thin films[19]. This cell incorporates a 9-pin glass-to-metal feedthrough, SAES getters and dispensers for doping and spotwelded or silver epoxied internal electrical connections. This doping cell can be thoroughly outgassed and provides a clean static vacuum environment for doping such that no parts of the cell are cold, so true equilibrium is established just as for the reflectivity and XRD samples and we can measure at low temperature without breaking vacuum. Resistivity was measured in the standard in-line 4-probe geometry.

Figure $2 \mathrm{~b}$ shows the temperature dependent resistivity of the same sample before outgassing, after outgassing and after partial $\mathrm{K}$ doping. The characteristic divergence at low temperature became 10 times more pronounced after outgassing while $\rho(300 \mathrm{~K})$ decreased somewhat. Upon $\mathrm{K}$ doping the resistivity drops rapidly at room temperature and $R(T)$ becomes essentially flat. While in this sample there is a remnant of the low-T divergence the total variation of $\mathrm{R}$ is only $20 \%$ in the full temperature range. All this points to the importance of both charge transfer and scattering in the explanation of the low-T divergence, but the present data is not sufficient to draw further conclusions.

We acknowledge financial support of NSF DMR97-30298, NSF-INT-9902050, NSFMTA-OTKA 021 (N31622), OTKA 32613. We thank Arthur F. Hebard for teaching us his unique doping technique for in-situ transport measurements.

\section{REFERENCES}

1. R. S. Lee, H. J. Kim, J. E. Fischer, A. Thess, and R. E. Smalley. Nature, 388:255, 1997.

2. L. Grigorian, G. U. Sumanasekera, A. L. Loper, S. Fang, J. L. Allen, and P. C. Eklund. Phys. Rev. $B, 58: \mathrm{R} 4195,1998$

3. A. M. Rao, P. C. Eklund, S. Bandow, A. Thess, and R. E. Smalley. Nature, 388:257, 1997.

4. T. Pichler, H. Kuzmany, H. Kataura, and Y. Achiba. Phys. Rev. Lett, 87:267401, 2001.

5. N. Bendiab, A. Righi, E. Anglaret, J. L. Sauvajol, L. Duclaux, and F. Beguin. Chem. Phys. Lett., 339:305, 2001.

6. Y. Iwasa, H. Fudo, Y. Yatsu, T. Mitani, H. Kataura, and Y. Achiba. Synth. Met., 121:1203, 2001

7. T. Pichler, M. Sing, M. Knupfer, M. S. Golden, and J. Fink. Solid State Commun., 109:721, 1999.

8. C. Bower, S. Suzuki, K. Tanigaki, and O. Zhou. Appl. Phys. A-Mater, 67:47, 1998.

9. L. Duclaux, K. Metenier, J. P. Salvetat, P. Lauginie, S. Bonnamy, and F. Beguin. Mol. Cryst. Liq. Cryst., 340:769, 2000.

10. B. Ruzicka, L. Degiorgi, R. Gaal, L. Thien-Nga, R. Bacsa, J. P. Salvetat, and L. Forro. Phys. Rev. B, 61:R2468, 2000.

11. E. Jouguelet, C. Mathis, and P. Petit. Chem. Phys, Lett., 318:561, 2000.

12. S. Kazaoui, N. Minami, R. Jacquemin, H. Kataura, and Y. Achiba. Phys. Rev. B, 60:13339, 1999.

13. A. S. Claye, N. M. Nemes, A. Janossy, and J. E. Fischer. Phys. Rev. B, 62:R4845, 2000.

14. A. G. Rinzler, J. Liu, H. Dai, P. Nikolaev, C. B. Huffman, F. J. Rodriguez-Macias, P. J. Boul, A. H. Lu, D. Heymann, D. T. Colbert, R. S. Lee, J. E. Fischer, A. M. Rao, P. C. Eklund, and R. E. Smalley. Appl. Phys. A-Mater, 67:29, 1998.

15. G. U. Sumanasekera, C. K. W. Adu, S. Fang, and P. C. Eklund. Phys. Rev. Lett., 85:1096, 2000

16. P. G. Collins, K. Bradley, M. Ishigami, and A. Zettl. Science, 287:1801, 2000.

17. A. Ugawa, A. G. Rinzler, and D. B. Tanner. Phys. Rev. B, 60:R11305, 1999.

18. D. J. Hornbaker, S. J. Kahng, S. Misra, B. W. Smith, A. T. Johnson, E. J. Mele, D. E. Luzzi, and A. Yazdani. Science, 295:828, 2002.

19. A. F. Hebard, M. J. Rosseinsky, R. C. Haddon, D. W. Murphy, S. H. Glarum, T. T. M. Palstra, A. P. Ramirez, and A. R. Kortan. Nature, 350:600, 1991. 